\title{
What's new for the clinician? Summaries of and excerpts from recently published papers
}

\section{The nonsurgical treatment of chronic periodontitis by means of scaling and root planning (SRP) with or without adjuncts: a systematic review and meta-analysis.}

\begin{abstract}
Smiley CJ, Tracy SL, Abt E, Michalowicz BS, John MT, Gunsolley J, Cobb CM, Rossmann J, Harrel SK, Forrest JL, Hujoel PP, Noraian KW, Greenwell H, Frantsve-Hawley J, Estrich C, Hanson N. The Journal of the American Dental Association; 2015; 146: $508-24$.

Clinicians are frequently challenged on the management of patients with periodontitis of varying extent and severity. Treatment options range from scaling and root planing (SRP) to SRP with adjunctive treatments, to surgical interventions. In 2011, the Council on Scientific Affairs of the American Dental Association (ADA) resolved to develop from a systematic review of the literature a clinical practice guideline for the nonsurgical treatment of chronic periodontitis with SRP with or without adjuncts. Clinical attachment level (CAL) was the sole outcome on which the reviewers compared the various treatments.
\end{abstract}

The study evaluated the following professionally applied or prescribed medical adjuncts: locally applied antimicrobials (chlorhexidine chips, doxycycline hyclate [DH] gel, and minocycline microspheres), nonsurgical use of lasers (diode, both photodynamic therapy [PDT] and non-PDT; neodymium:yttrium-aluminum-garnet [Nd:YAG]; and erbium), systemic antimicrobials, and systemic subantimicrobial-dose doxycycline (SDD). They also considered systemic antimicrobials and systemic SDD separately because the latter appears to inhibit mammalian collagenase activity (matrix metalloproteinase 8) and not function as an antibiotic. The review team did not consider experimental adjuncts, adjuncts not currently available in the United States, nonprescription (over-the-counter) adjuncts, or surgical treatments.

The following clinical questions were reviewed:

- Question 1: In patients with chronic periodontitis, does SRP (hand or ultrasonic), when compared with no treatment, supragingival scaling and polish

Jeff Yengopal: BChD, MChD (Community Dentistry) Stellenbosch. Department of Community Dentistry, School of Oral Health Sciences,

University of the Witwatersrand. E-mail: Veerasamy.Yengopal@wits.ac.za.

\author{
ACRONYMS \\ ADA: American Dental Association \\ CAL: clinical attachment level \\ DH: doxycycline hyclate \\ Nd:YAG: neodymium:yttrium-aluminum-garnet \\ PD: photodynamic therapy \\ SD: subantimicrobial-dose doxycycline \\ SRP: $\quad$ scaling and root planing
}

(prophylaxis), or debridement, result in greater improvement of CAL?

- Question 2: In patients with chronic periodontitis, does the use of adjuncts such as local antibiotics or antimicrobials, systemic antibiotics, combinations of local and systemic antibiotics, agents for biomodification, host modulation or nonsurgical lasers, result in a greater improvement of CAL, compared with that achieved with SRP alone,?

\section{METHODS}

A comprehensive search strategy was developed and two electronic databases (PubMed and Embase) and the reference section of selected systematic reviews were screened to identify missed references. The search was first conducted in October 2012 and updated in July 2014. Randomized controlled trials (both parallel-arm and splitmouth studies) were included if they were published after 1960, written in English, and reported changes in $\mathrm{CAL}$ at least six months after randomization. Clinical attachment level (CAL) was chosen as a primary outcome because probing depth changes fail to capture the effect of nonsurgical treatment. Excluded were studies of aggressive periodontitis, as well as studies in which the adjunct was administered more than one week after SRP or was reapplied to progressing (worsening) tooth sites. The authors independently reviewed and extracted the relevant data from included studies and appraised each study with the Cochrane Risk of Bias Tool. In assessing the effectiveness of SRP alone (Question 1), the reviewers compared mean change in CAL between 
SRP and controls. To assess adjuncts (Question 2), the mean changes between groups receiving SRP and those receiving SRP plus an adjunct were compared. Metaanalyses was done by using the random effects model. Overall results for each treatment or adjunct was reviewed and the level of certainty in the evidence was assessed as high, moderate, or low as shown in Table 1 below.

\section{SUMMARY OF INTERVENTIONS, STATISTICAL SIGNIFICANCE $(P<0.05$ OR $P>0.05)$ AND STRENGTH OF EVIDENCE.}

Scaling and root planing (SRP) versus no treatment Eleven studies met the inclusion criteria for reporting the effect of SRP compared with no treatment, supragingival scaling, or debridement, on chronic periodontitis. Compared with no treatment, SRP treatment resulted in an average of 0.49 -millimeter gain in CAL $(95 \%$ confidence interval [Cl], 0.36-0.62 mm; $\mathrm{p}<0.05)$. Overall level of certainty in the evidence was judged to be moderate.

Systemic subantimicrobial-dose doxycycline (SDD) and SRP

SDD (Periostat, CollaGenex Pharmaceuticals) is considered a host-modulating agent. Specifically, it inhibits the collagen-degrading enzymes of the host. Compared with SRP alone, SRP plus SDD resulted in a 0.35-mm mean gain in CAL (95\% Cl, 0.15-0.56; $p<0.05$ ).
Overall level of certainty in the evidence was judged to be moderate.

\section{Systemic antimicrobials and SRP}

Twenty-four studies met the inclusion criteria for reporting the effect of SRP plus a systemic antimicrobial versus SRP alone. All were parallel-group trials.

Compared with SRP alone, SRP plus systemic antimicrobials resulted in a $0.35-\mathrm{mm}$ mean gain in CAL (95\% Cl, 0.20-0.51, p < 0.05). Overall level of certainty in the evidence was judged to be moderate.

\section{Locally delivered antimicrobials and SRP}

\section{Chlorhexidine chips and SRP}

Compared with SRP alone, SRP plus chlorhexidine chips resulted in a $0.40-\mathrm{mm}$ mean gain in $\mathrm{CAL}(95 \% \mathrm{Cl}, 0.24$ 0.56 ; $p<0.05)$. Overall level of certainty in the evidence was judged to be moderate.

\section{Doxycycline Hyclate (DH) gel and SRP}

Three small studies met the inclusion criteria for reporting the effect of SRP plus the local delivery of $\mathrm{DH}$ gel compared with SRP alone. Two were split-mouth studies and one study was a parallel-group trial. Compared with SRP alone, SRP plus DH gel resulted in a 0.64-mm mean gain in CAL (95\% Cl, 0.00-1.28; p> 0.05). Overall level of certainty in the evidence was judged to be low.

Table 1: Level of certainty of the evidence

\begin{tabular}{|c|c|}
\hline $\begin{array}{l}\text { Level of certainty in } \\
\text { effect estimate }\end{array}$ & Description \\
\hline High & $\begin{array}{l}\text { The body of evidence usually includes consistent results from well-designed, well-conducted studies } \\
\text { in representative populations. This conclusion is unlikely to be affected strongly by the results of future } \\
\text { studies. } \\
\text { This statement is established strongly by the best available evidence. }\end{array}$ \\
\hline Moderate & $\begin{array}{l}\text { As more information becomes available, the magnitude or direction of the observed effect could change, } \\
\text { and this change could be large enough to alter the conclusion. } \\
\text { This statement is based on preliminary determinations from the current best available evidence, but } \\
\text { confidence in the estimate is constrained by one or more factors, such as the following: } \\
\text { - } \quad \text { Limited number or size of studies } \\
\text { - } \quad \text { Plausible bias that raises some doubt about the results } \\
\text { - Inconsistency of findings across individual studies } \\
\text { - Imprecision in the summary estimate } \\
\text { - } \quad \text { Limited applicability because of the populations of interest } \\
\text { - } \quad \text { Lack of coherence in the chain of evidence }\end{array}$ \\
\hline Low & $\begin{array}{l}\text { More information could allow a reliable estimation of effects on health outcomes. } \\
\text { The available evidence is insufficient to support the statement, or the statement is based on extrapolation } \\
\text { from the best available evidence. The evidence is judged to be insufficient, or the reliability of estimated } \\
\text { effects is limited by factors such as the following: } \\
\text { - } \quad \text { Limited number or size of studies } \\
\text { - } \quad \text { Plausible bias that seriously weakens confidence in the results } \\
\text { - Inconsistency of findings across individual studies } \\
\text { - Imprecision in the summary estimate } \\
\text { - } \quad \text { Gaps in the chain of evidence } \\
\text { - } \quad \text { Findings not applicable to the populations of interest } \\
\text { - } \quad \text { Evidence of publication bias } \\
\text { Lack of information on important health outcomes }\end{array}$ \\
\hline
\end{tabular}




\section{Minocycline microspheres and SRP}

Compared with SRP alone, SRP plus minocycline microspheres resulted in a $0.24-\mathrm{mm}$ mean gain in CAL (95\% Cl, -0.06 to $0.55 ; p>0.05)$. Overall level of certainty in the evidence was judged to be low.

\section{Nonsurgical use of lasers and SRP}

Several types of lasers are used nonsurgically as adjunctive treatments with SRP. The lasers are categorized primarily by the wavelength of the emitted light. Five categories of lasers are included and described here.

\section{PDT ( photodynamic therapy) diode laser and SRP} Compared with SRP alone, SRP plus PDT diode laser resulted in a $0.53-\mathrm{mm}$ mean gain in CAL $(95 \% \mathrm{Cl}, 0.06-$ 1.00; $p<0.05)$. Overall level of certainty in the evidence was judged to be moderate.

\section{Non-PDT diode laser and SRP}

Compared with SRP alone, SRP plus non-PDT diode laser resulted in a $0.21-\mathrm{mm}$ mean gain in CAL $(95 \% \mathrm{Cl},-0.23$ to $0.64 ; p>0.05)$. Overall level of certainty in the evidence was judged to be low.

\section{Nd:YAG laser and SRP}

Compared with SRP alone, SRP plus Nd:YAG laser resulted in a $0.41-\mathrm{mm}$ mean gain in CAL $(95 \% \mathrm{Cl},-0.12$ to $0.94 ; p>0.05)$. Overall level of certainty in the evidence was judged to be low.

\section{Erbium laser and SRP}

Compared with SRP alone, SRP plus erbium laser resulted in a 0.18- $\mathrm{mm}$ mean gain in $\mathrm{CAL}(95 \% \mathrm{Cl},-0.63$ to $0.98 ; p>0.05)$. Overall level of certainty in the evidence was judged to be low.

Summary statements on nonsurgical use of lasers Unlike other instruments, lasers have no defined and accepted protocols for standard usage. Because every operator determines his or her own protocol on the basis of anecdotal rules or experiences, the potential for adverse events to the tooth and patient is higher than it is with other local delivery systems. Also, laser wavelengths vary, affecting the hard and soft tissues differently, and making comparisons between lasers unpredictable and often incorrect. Common protocols are needed for each laser used in the nonsurgical therapy of chronic periodontitis to allow for repeatable results and comparisons among studies in the literature. The wide ranges found in the few studies considered for CAL gain or loss demonstrate the need for larger sample sizes and for additional studies, properly to evaluate the potential benefits of laser use as an adjunct to SRP. At this time, on the basis of the criteria set in this systematic review evidence-based literature, there is in fact insufficient evidence for any laser wavelength except that of the PDT diode lasers to enable accurate definition of the benefits of adjunctive nonsurgical therapy of periodontitis.

\section{CONCLUSIONS}

On average, treatment of chronic periodontitis with SRP was associated with a $0.5-\mathrm{mm}$ improvement in CAL against no treatment at a moderate level of certainty.
The reviewers found benefits in four adjunctive therapies as compared with SRP alone: systemic SDD, systemic antimicrobials, chlorhexidine chips, and PDT with a diode laser at a moderate level of certainty. They found a low level of certainty on the benefits of the other five adjunctive therapies. Combinations of SRP with these assorted adjuncts resulted in a range of average CAL improvements between 0.2 and $0.6 \mathrm{~mm}$ over SRP alone.

\section{IMPLICATIONS FOR PRACTICE}

This high quality review, which is used as an ADA (American Dental Association) guideline, should serve as an important evidence base for clinicians considering one or more of the above interventions.

\section{Reference}

1. Smiley CJ, Tracy SL, Abt E, Michalowicz BS, et al. Systematic review and meta-analysis on the nonsurgical treatment of chronic periodontitis by means of scaling and root planing with or without adjuncts. The Journal of the American Dental Association; 2015: 146: 508 - 524.e5. 